\title{
A Complication of Surgical Abortion: A Rare Presentation
}

\author{
${ }^{1}$ Manjeet Kaur, ${ }^{2}$ Dilpreet Kaur Pandhar, ${ }^{3}$ Reeti Mehra, ${ }^{4}$ Anju Huria
}

\begin{abstract}
Dilatation and evacuation ( $D$ and $E$ ) is the most commonly used method for later abortion. It is one of the safest procedure when performed by experienced personnel. Complication rates are high in second trimester abortion compared to first trimester. Rupture of uterine wall covered with organized blood clots is very rare complication after second trimester surgical abortion. It is a surgical emergency and may have deleterious consequences if diagnosis is delayed.
\end{abstract}

Keywords: Dilatation and evacuation, Second trimester abortion, Rupture of uterus.

How to cite this article: Kaur M, Pandhar DK, Mehra R, Huria A. A Complication of Surgical Abortion: A Rare Presentation. J South Asian Feder Obst Gynae 2014;6(1):33-34.

Source of support: Nil

Conflict of interest: None

\section{INTRODUCTION}

Dilatation and evacuation ( $\mathrm{D}$ and $\mathrm{E}$ ) is a common ${ }^{1}$ and relatively safe gynecological procedure if done by experienced hands. ${ }^{2,3}$ Risk of complications increases with gestational age. ${ }^{2}$ Common complications after second trimester surgical abortion are incomplete abortion, cervical laceration, hemorrhage, uterine perforation and infection. ${ }^{1}$ Uterine rupture is reported with second trimester medical abortion but, it is very rare after surgical abortion. ${ }^{1}$ In a tertiary care center, patients may be referred late as septic abortion with septicemia, hemoperitoneum, gut injury, peritonitis, etc. but, we had a patient with this rare presentation in which diagnosis is delayed and condition terminated into subtotal hysterectomy.

\section{CASE REPORT}

A 25 years female P2L1A1 with previous one cesarean section was referred to the hospital for vaginal bleeding after $\mathrm{D}$

\footnotetext{
${ }^{1}$ Senior Resident, ${ }^{2}$ Assistant Professor, ${ }^{3}$ Associate Professor, ${ }^{4}$ Professor and Head

${ }^{1-4}$ Department of Obstetrics and Gynecology, Government Medical College and Hospital, Chandigarh, India
}

Corresponding Author: Manjeet Kaur, Senior Resident Department of Obstetrics and Gynecology, Government Medical College and Hospital, Sector 32, Chandigarh, India e-mail: drmanjeetkaur99@gmail.com and $\mathrm{E}$ for missed abortion. Two days back, she was admitted in private nursing home for vaginal bleeding. After diagnosing missed abortion of about 20 weeks on ultrasound scan $\mathrm{D}$ and $\mathrm{E}$ was performed there.

According to patient she conceived in lactation amenorrhea and was probably having 5 months period of gestation for which no formal antenatal checkup was done before.

As per her obstetrics history-Her married life was 4 years. She was P2L1A1. First delivery was at home and baby was stillborn of unknown cause. Second delivery was full term lower segment cesarean section 1 year back for meconium stained liquor with no postoperative complication.

General physical examination showed significant pallor with normal systemic examination. Pulse was 106/min and blood pressure was 100/68 mm Hg. Abdominal examination reveals 16 to 18 weeks size uterus, soft abdomen with pfanenstiel scar and no scar tenderness. Vaginal examination revealed vaginal bleeding with soft cervix, posterior OS closed, lower segment ballooned up and 16 to 18 weeks size uterus.

Patient had 2 to 3 fainting episodes after admission. All routine investigations were within normal limits except low hemoglobin (6.4 gm\%). Foleys catheter inserted. On transabdominal ultrasound no fetal tissue and no free fluid seen in uterine cavity and peritoneal cavity respectively. In lower uterine segment homogenous mass is seen. Diagnosis of uterine fibroid with complete abortion associated with anemia was made provisionally and patient treated conservatively with blood transfusion. On third day, patient became hemodynamically unstable. Her hemoglobin dropped to $4.7 \mathrm{gm} \%$ and urine become blood stained. Her pulse rate increased to $126 / \mathrm{min}$ and blood pressure dropped to $88 / 60$ $\mathrm{mm} \mathrm{Hg}$. Repeat ultrasound scan shows $5.5 \times 8 \mathrm{~cm}$ collection inside uterine cavity with $8.3 \times 9 \mathrm{~cm}$ hypoechoeic area in continuation of anterior wall of uterus and extension in area of broad ligament on right side. In Doppler study, there was no flow observed in the mass. Possibility of scar perforation with blood collection at scar site made and patient scheduled for exploratory laparotomy. After resuscitation midline infraumblical incision given under general anesthesia. Uterovesical fold was distended with blood clots (about 1.5 l). Uterovesical peritoneum opened and blood clots were evacuated. Anterior uterine wall was ruptured and replaced by blood clots which were also filling the uterine cavity and 


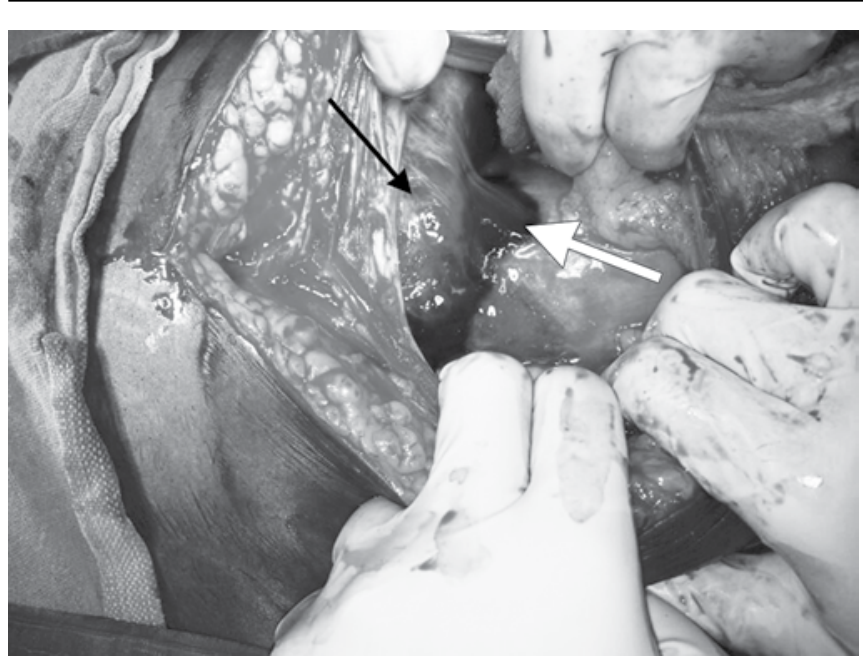

Fig. 1: Rupture of uterine wall (shown by thick arrow) with right broad ligament hematoma (depicted by thin arrow)

extended into right broad ligament (Fig. 1). All blood clots were evacuated. Anterior uterine wall was dehiscent almost up to anterior vaginal fornix so repair was not possible. Subtotal hysterectomy done, bladder integrity checked and drain kept. Three units of blood transfused intraoperatively. Postoperative period was uneventful and patient discharged in a satisfactory condition on 5 th postoperative day.

\section{DISCUSSION}

Dilation and evacuation is one of the common method of surgical abortion in second trimester. ${ }^{1}$ Though risk of complication is low, it increases with gestational age. ${ }^{2}$ Common complications after second trimester surgical abortion are incomplete abortion (retained products of conception), cervical laceration, hemorrhage, uterine perforation and infection. Uterine rupture is very rare.

Uterine perforation just above the internal cervical os may cause hemorrhage into the broad ligament. ${ }^{4}$ Lateral uterine perforation can lead to intraperitoneal hemorrhage and broad ligament hematoma. ${ }^{5}$ Such patients report back pain, fullness or pressure in the anorectal area or an urge to push but no such type of symptoms present in our patient. Although history of fainting episodes and anemia was present.

In our patient, organized intrauterine hemorrhage and no free fluid in uterine cavity leads to diagnosis of uterine fibroid with complete abortion at first but later worsened patient condition and new ultrasonographic findings change our management.

Diagnosis of such condition is difficult and delayed where common causes of bleeding per vaginum like retained products of conception, cervical laceration and infection, etc. were not found after surgical abortion. This presentation of anterior uterine wall rupture with organized blood clots in uterine cavity extending into the right broad ligament after surgical abortion is very rare and is a potentially life-threatening condition. Early suspicion of such condition and emergency exploratory laparotomy is the solution for such circumstances.

\section{REFERENCES}

1. Grossman D, Blanchard K, Bluementhal P. Complications after second trimester surgical and medical abortion. Reproductive health matters 2008;16:173-182.

2. Diedrich J, Steinauer J. Complications of surgical abortion. Clin Obstet Gynecol 2009;52:205-212.

3. Koshiba A, Koshiba H, Noguchi T, Iwasaku K, Kitawaki J. Uterine perforation with omentum incarceration after dilatation and evacuation/curettage: magnetic resonance imaging findings. Arch Gynecol Obstet 2012;285:887-890.

4. Taylor DS, Jurow R. Complications of surgical abortions. Management of common problems in obstetrics and gynecology. In: Goodwin TM, Montoro MN, Muderspach L, Paulson R, Roy S, editors. Chichester, Wiley Blackwell 2010.517.

5. Abrams YM. Dilatation and Curettage. The essential guide to primary care procedures. In: Mayeaux EJ, editor. Philadelphia, Lippincott Williams and Wilkins 2009;p.554. 